\title{
The Study of Usage of Hyperledger Fabric in Agricultural Ecommerce
}

\author{
Vivek Ray ${ }^{\mathrm{a}, 1}$, Anmol Singh ${ }^{\mathrm{a}}$, Mayank Singh ${ }^{\mathrm{a}}$, Rishi Singh ${ }^{\mathrm{a}}$, and Dr. Shushila Palwe ${ }^{\mathrm{b}}$ \\ a Student, Dr. Vishwanath Karad MIT World Peace University, Pune 411047, India \\ ${ }^{b}$ Assistant Professor, School of CET, Dr. Vishwanath Karad MIT World Peace \\ University, Pune 411047, India
}

\begin{abstract}
With the advent of COVID-19, the ecommerce industry in India has seen an inflection point with rise in the demand across all the segments of the industry. Much ecommerce has been started to cater to the supply and demand mismatch in agricultural goods front. In these times, Blockchain is seen to create a trust bridge for all the stakeholder to transact through goods and supplies with minimal risks involved. But, to use this technology the technical, economical and a scalable approach to this technology is still a very prominent requirement for mass adoption. Permissionless Blockchain build on Proof of Work consensus protocol cannot be used due to their slow speed, low scalability, and high energy consumption for network functioning. This paper concludes with the possibility of using a Permissioned blockchain such as Hyperledger Fabric to not only solve the several underlying issues to facilitate efficiency in the Ecommerce architecture, with critical view on the mass adoption.
\end{abstract}

Keywords. Ecommerce, Blockchain, Permissionless Blockchain, Proof of Work, Permissioned Blockchain, Hyperledger Fabric.

\section{Introduction}

Ecommerce is a term given to illustrate electronic commerce, which refers to goods and supplies being sold over the internet through channels such as websites and mobile applications. Due to low commutability in COVID-19, the inter-net landscape through mobile applications, experienced a surge in the ecommerce industry because of high demand of goods [1]. But the growth of ecommerce business in agriculture sector is still not matching with all the stakeholder, as the profits are highly concentrated towards the middle parties involved in the whole distribution as well as marketing of the goods. This challenge can be fixed by creating an infrastructure of ecommerce with trust reinstated to all the stakeholders with the help of use of digital distributed ledger in blockchain. A blockchain is a platform of decentralized computation and information sharing platform that enables multiple authoritative domains, who do not trust each other, to cooperate, coordinate and collaborate in a rational decision-making process. Individual transaction data files, called as blocks, are managed through specific software

\footnotetext{
${ }^{1}$ Vivek Ray, Dr. Vishwanath Karad MIT World Peace University, Pune Email: vivekray903@gmail.com.
} 
platforms that allow the data to be transmitted, processed, stored, and represented in human readable form. This digital ledger forms the foundation for trust in a trustless digital environment. The usage of blockchain network can be seen through its practical application in several cryptocurrencies [2] which are its most dominant usage. In agricultural ecommerce, the blockchain offers to help in different operations such as in traceability, supervision, and management of goods.

The initial blockchain platform such as Bitcoin and Ethereum were permissionless blockchain [3] as they were open to participation by nature and were usually used in application involving multidisciplinary use cases that required trustworthy and decentralized execution of business logic. But with the benefits, permissionless blockchain also suffered from several drawbacks such as (i) low performance because of high throughput of transaction requests, and (ii)confidentiality issues. These drawbacks cause decrease in efficiency and hence, create problem for the utilization of public blockchains by large businesses since these problem prevent the integration of enterprise grade systems that usually require secure, high performance applications that guarantee reliable and consistent transactions to a volatile public network. Therefore, permissioned blockchains were developed for avoiding these shortcomings.

Permissioned blockchain are blockchain networking systems in which the participation in the network and in the transactions of the network required explicit authorization from the administrators of the network. Permissioned blockchains are suitable for competing enterprises which in out cases revolves around the various Ecommerce platform that are, nonetheless, willing to engage in collaborative processes without employing third parties, such as notaries, or centralized settlement networks. There are several factors to prove that permissioned blockchains provide various enhancements over their permissionless counterparts, such as: (i) the participation in the blockchain is limited to a specific group of users that requires explicit system reconfiguration to be modified, permissioned blockchains use Byzantine Fault Tolerant (BFT) protocols, which are a better alternative in terms of transaction latency and throughput. (ii) Furthermore, permissioned blockchains are generally better in terms of data privacy and data sharing as sensitive transaction can be secured from access,(iii) Finally, most permissioned blockchain systems are directed toward transaction finality as well as other transactional properties which are not focused by public blockchain.

\section{Literature Review}

In the article of Kamilaris and others [4], the solution suggested through blockchain were: (i)traceability in value chains, (ii) participation of small farmers, (iii)fair pricing through whole value chain, (iv)more informed consumer purchasing decision, (v)reduced transaction fees and less dependence on intermediaries, and (vi) more transparent transaction and less frauds.

As suggested by V.S. Yadav and A. R. Singh [5], blockchain based mobile app have the ability to solve the above listed problems of agriculture, however the proposed solution does also have the problems as suggested by Kamilaris[4]: (i) High uncertainties and market volatility of transaction fees, (ii) No regulation in place , (iii)Scalability issues due to latency of transactions,(iv)Privacy issues, and (v)decline of cryptocurrencies prices may lead to decline in growth of block-chain network or even making the whole network out of order. 
As Hyperledger Fabric fixes these issues with a predominant permissioned architecture which not only suits the commercial needs but also the business privacy involved, thus it plays a better role for the supported blockchain requirements involved for Ecommerce needs.

\subsection{Hyperledger Fabric Overview}

Hyperledger Fabric, an open-source project initiated by Linux Foundation and IBM, is the modular blockchain framework which provides de facto standard for enterprise blockchain platforms. It has provided a foundation for developing enterprise-grade applications and industry solutions, the open, modular architecture that uses plug-andplay components to be applied for several use cases [6].

Fabric executes distributed applications written in general-purpose programming languages like Go, Java or Node.js. There are three stages in Fabric Blockchain operation: Execution, Ordering and Validation [7]. Fabric Network securely monitors the execution operation in such a way that the data structure can only be updated without any inherent transaction fees as it has no cryptocurrency built in for the network. Fabric consists of two main parts:

In Hyperledger Fabric, smart contract is referred as chain code, which is program code that implements the application logic and runs during the execution phase. It is one of the integral parts of the distributed application hosted on Fabric Blockchain.

An endorsement policy is a static library for transaction validation during validation phase in Fabric Blockchain, which is instructed by the chain code. As the Fabric Blockchain is permissioned network, so only the designated administrators have the permission to modify system policies that are used in during node designation by the Membership Service Provider (MSP).

.A Fabric blockchain consists of a set of nodes that form a network (Fig. 1). All nodes that participate in the network have an identity, as provided by a modular MSP. MSP has three roles which handle the overall architecture of the network:

- Clients: These are the applications which helps the person to initiate the transactions on network. They have SDK packages which is connection towards the Chaincodes hosted on Fabric Blockchain.

- $\quad$ Peers(P): Peers maintain the state of the network and the copy of ledger. All peers commit blocks to the distributed ledger. In Fabric Blockchain there are two special types of peers:

- Endorsing Peer: Peers with chaincode installed which stores transaction of chaincodes in an isolated chaincode containers and prepares proposal based on results of chaincode. They produce endorsement signature and simulation of transaction. This signature is sent back to client.

- Committing Peers: Peers with only purpose of maintaining and updating their full ledger of records. They do not have any association with chaincodes or their functions.

- Ordering Service Nodes (OSN): The ordering service nodes accept verified transaction, orders them into a block, and delivers the blocks to the committing peers. 


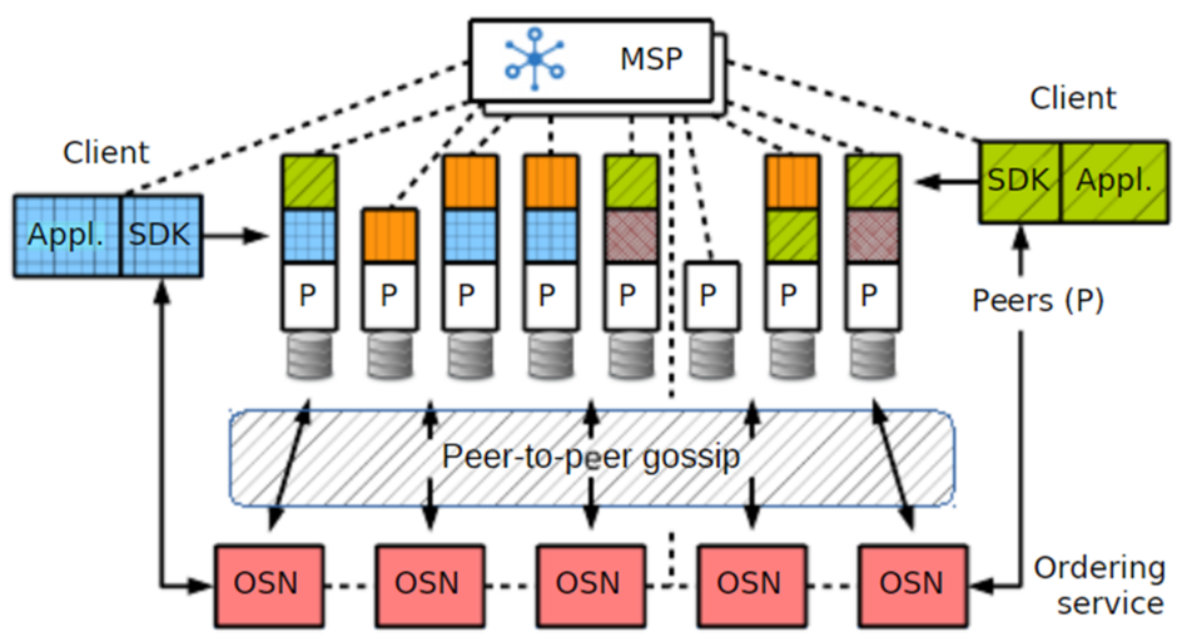

Figure 1. A Hyperledger Fabric Blockchain Network

All these components form the application structure required to operate the overall business processes which will write the data onto the Fabric Blockchain. The process starts with the client sending a transaction to Endorsing peer. Endorsing peers sends back a signature on the valid transaction. Figure 1 this signature transaction is sent to OSN where it is verified. After verification, this transaction is forwarded from OSN to all the committing peers via Peer-to-Peer gossip. This gossip enables the transaction between two parties to be propagated to all the committing peers for maintaining the record of transaction in distributed ledgers of Fabric Blockchain Network.

\section{Proposed Model for Ecommerce}

Using the Hyperledger Fabric architecture, the proposed model uses chaincode which is a container for deploying Smart contract on Fabric network integrated with mobile application running on smartphones. One or several smart contracts for different functionalities can be defined within a chaincode involved in Ecommerce. Every smart contract has a name that is uniquely identified in a chaincode. Chaincode is executed within a secured Docker container isolated from the endorsing peer process. It initializes and manages ledger state through transactions submitted by application. The chaincode can address the smart contract for:

- Authentication: This smart contract can address to the issue of authenticating the seller through pre-defined channel as the regulatory governmental bodies in-scribe. This insures the services reachability to the marginalized/nonmarginalized farmers with the required Consumer demand.

- Traceability: The factor of traceability is based on the process of the goods transaction as well as the risk involved in case of damaged/spoiled goods. The logistical approach of Hyperledger Fabric in the first scenarios is based on the parties being exchanging the goods on a step and creating changes in the network based on the future step till the goods reaches to the final stakeholder. 
In the second scenario, since the data obtained from authentication makes the process of origin of the goods involved traceable, in case of any food hazard involved can further reported to the regulatory.

- Transaction Records: The transaction record of the mutual exchange of goods with the financial data can be further pushed to the smart contract which would deal with the data being added to the chain of records in the ledger history of both the parties. This would make both the parties being adhered to the goods being exchanges between them as per the acknowledged terms in all aspect.

\section{Limitations}

The Agricultural Ecommerce platform cater to the demand of the buyer as well as supply of the farmer. However, the Fabric platform's sustainability also depends on the number as well as the volume of transactions involved. This factor makes the usability of this technology being more concentrated toward bigger corporation or ecommerce platforms where transaction is of higher volume or higher value. The correlation of using more transparency in the platform for having higher trust is debatable based on notions of the entire end stakeholder (Farmers and Buyer) in the whole value chain. Thus, for transparency on the transaction level, where both the parties are assured of successful transaction through application is the prime priority. Blockchain can only add more value and trust if already existing transaction chain is successfully implemented which can make Fabric network optimal for use.

\section{Result}

On the aspects of the operational as well as financial efficiency, the permissioned blockchain could cater all the functionalities of permissionless blockchain without any issue in terms of transactional latency as well as the cost per transaction. Privacy and transparency are equally important balancing outlook for an ecommerce which depends on the platform developers as to what parameter of a transaction needed to be declared without having a breach in the involved trade information. As per the historical volumes in Agricultural Ecommerce of India, permissioned blockchain could scale to the needs, with providing all the essential required functionalities of a required immutable ledger for the stakeholders in the supply and demand chain. The proposed model distributes workloads of the functionalities involving the authentication, traceability, and transaction record management. These systematic processes can change based on the geographical as well as applicational functionalities to be achieved for stakeholders.

The systematic processes can be explained by the Figure 2 is an example of a vegetable cargo, which is transported from buyer to seller through four steps: placement of order with successful monetary transaction, its acceptance from seller, its delivery and successfully reception from buyer's end. These changes are made to the block of data in Fabric which can only get updated but in its entirety, transactions cannot be deleted. Thus, the integrity of whole transaction is maintained without any 
role of middle party in the entire process except the optimal working of the systems involved.

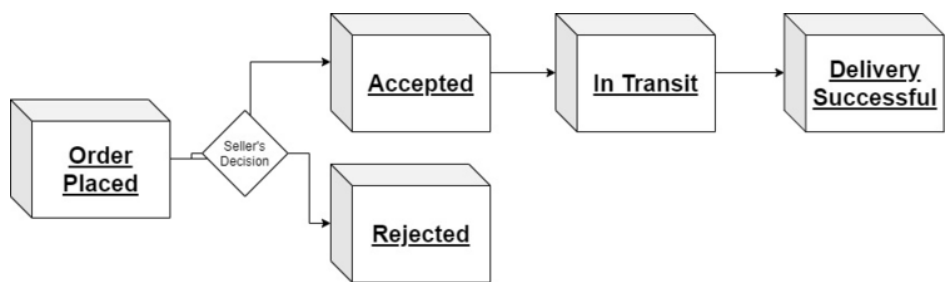

Figure 2. Block changes in Fabric on "Status" variable after every step in Agricultural Ecommerce

\section{Conclusion}

The proposed model could create a systemic channel for the Ecommerce platform to exchange goods between buyer and farmer. However, these outcomes are also based on the foundational foothold of a platform before its usability of Hyperledger Fabric Blockchain network. The simplified Graphical User Interface as well as dominancy of the Ecommerce application in the region will help this feature towards being accepted and Fabric being mass adopted in other sectors of Ecommerce.

\section{References}

[1] Shrey Nougarahiya, Gaurav Shetty, Dheeraj Mandloi, F.: A Review of E - Commerce in India: The Past, Present, and the Future, In: Research Review International Journal of Multidisciplinary. Volume 06 Issue 03, 12-22 (2021).

[2] David Vidal-Tomás,T.: Transitions in the cryptocurrency market during the COVID-19 pandemic: A network analysis, In.: Finance Research Letters (2021)

[3] G. Falazi, M. Hahn, U. Breitenbücher, F. Leymann and V. Yussupov, T.: Process-Based Composition of Permissioned and Permissionless Blockchain Smart Contracts, In.: IEEE 23rd International Enterprise Distributed Object Computing Conference (EDOC), Paris, France, pp. 77-87(2019).

[4] Andreas Kamilaris, Agusti Fonts, Francesc X. Prenafeta-Boldv́, F.: The rise of blockchain technology in agriculture and food supply chains, T.:Trends in Food Science \& Technology,Volume 91, Pages 640652(2019)

[5] Vinay Surendra Yadav, and A. R. Singh, , F.: Use of blockchain to solve select issues of Indian farmers, In: AIP Conference Proceedings 2148, 030050 (2019)

[6] IBM (What is Hyperledger Fabric?), https://www.ibm.com/in-en/topics/hyperledger,last accessed 2021/11/04.

[7] Elli Androulaki, Artem Barger, Vita Bortnikov, Christian Cachin, Konstantinos Christidis, Angelo De Caro, David Enyeart, Christopher Ferris, Gennady Laventman, Yacov Manevich, Srinivasan Muralidharan, Chet Murthy, Binh Nguyen, Manish Sethi, Gari Singh, Keith Smith, Alessandro Sorniotti, Chrysoula Stathakopoulou, Marko Vukolić, Sharon Weed Cocco, and Jason Yellick. T.: Hyperledger fabric: a distributed operating system for permissioned blockchains. In.: Proceedings of the Thirteenth EuroSys Conference. Association for Computing Machinery, New York, NY, USA, Article 30,1-15(2018) 